\title{
INCENTIVOS GOVERNAMENTAIS EM PPP: UMA ANÁLISE POR OPÇÕES REAIS
}

\author{
GOVERNMENTAL INCENTIVES IN PPP: AN ANALYSIS WITH REAL OPTIONS \\ INCENTIVOS GUBERNAMENTALES EN APP: UN ANÁLISIS POR OPCIONES REALES
}

\section{RESUMO}

Em novembro de 2005, o Governo do Estado lançou edital de licitação para serviços de transporte de passageiros da Linha 4 do Metrô de São Paulo. Neste trabalho, utilizamos a metodologia de opções reais para modelar o impacto dos incentivos governamentais existentes no contrato de concessão sobre o valor do projeto, determinar o grau de redução de risco obtido e o seu custo para o Estado. Os resultados indicam que estes incentivos são eficazes em reduzir o risco do projeto e aumentam o seu resultado em 36\%, a um custo para o Estado de 5\% do valor total do projeto. Adicionalmente, analisamos também a relação custo/ benefício para o Estado de modelos alternativos de mitigação de risco e mostramos que, sem aumentar o custo para o Governo, a eficácia desse mecanismo pode ser incrementada aumentando-se o peso da parcela de garantia de demanda em relação à parcela de contraprestação.

PALAVRAS-CHAVE Parceiras público-privadas, desenho de incentivos, garantia de demanda, opções reais, avaliação de projetos.

Luiz Eduardo Teixeira Brandão brandao@iag.puc-rio.br

Professor do Departamento de Administração, Pontifícia Universidade Católica do Rio de Janeiro - Rio de Janeiro - RJ, Brasil

Carlos de Lamare Bastian-Pinto carbastian@gmail.com

Professor do Programa de Mestrado Acadêmico, Universidade do Grande Rio - Rio de Janeiro - RJ, Brasil

Leonardo Lima Gomes leonardolima@iag.puc-rio.br

Professor do Departamento de Administração, Pontifícia Universidade Católica do Rio de Janeiro - Rio de Janeiro - RJ, Brasil

Marina Schuabb Salgado marinaschuabb@hotmail.com

Coordenadora Financeira do Farol Apoio Marítimo - Rio de Janeiro - RJ, Brasil

Abstract On November 2005 the State Government of São Paulo, Brazil, announced the intention to bid a contract to provide passenger services for the Line 4 of the São Paulo Metropolitan Subway System. In this article we use the real options approach to analyze the effect of the government guarantees provided in the contract on the value and the risk of the project, as well as the cost and the risk of these guarantees to the government. The results indicate that the incentives proposed are effective in reducing the risk and increase the net value of the Project by $36 \%$ at a cost to the State of only $5 \%$ of the total value of the project. Additionally, we show that for a given cost to the government, the most effective risk reduction mechanisms are the ones that include a higher portion of demand guarantees in relation to the investment subsidy.

Keywords Public-private partnership, design of incentives, demand guarantees, real options, valuation.

Resumen En noviembre de 2005, el Gobierno del Estado lanzó un edicto de licitación para servicios de transporte de pasajeros de la Línea 4 del Metro de São Paulo. En este trabajo, utilizamos la metodología de opciones reales para modelar el impacto de los incentivos gubernamentales existentes en el contrato de concesión sobre el valor del proyecto, determinar el grado de reducción de riesgo obtenido y su costo para el Estado. Los resultados indican que estos incentivos son eficaces para reducir el riesgo del proyecto y aumentan su resultado en 36\%, a un costo para el Estado del $5 \%$ del valor total del proyecto. Adicionalmente, analizamos también la relación costo-beneficio para el Estado de modelos alternativos de mitigación de riesgo y mostramos que, sin aumentar el costo para el Gobierno, la eficacia de ese mecanismo puede ser incrementada aumentando el peso de la cuota de garantía de demanda en relación a la cuota de contraprestación.

Palabras clave Aliadas Público-Privadas, diseño de incentivos, garantía de demanda, opciones reales, evaluación de proyectos. 


\section{INTRODUÇÃo}

Em novembro de 2005, o Governo do Estado de São Paulo lançou o Edital no 42325.212 para a construção e exploração da operação, por 30 anos, dos serviços de transporte de passageiros da Linha 4 do Metrô, também conhecida como Linha Amarela. O projeto envolvia investimentos totais de $\mathrm{R} \$ 3,34$ bilhões de reais e consistia em um trecho com $12,8 \mathrm{~km}$ de extensão e 11 estações de passageiros conectando o centro da cidade ao extremo oeste da capital. Além de servir para descongestionar outros ramais viários, a Linha 4 teria, ainda, a importante função de interligar os 50,3 $\mathrm{km}$ de vias metroviárias existentes, distribuídas em quatro linhas, ao sistema ferroviário paulista.

Os primeiros estudos para a construção do Metrô de São Paulo foram realizados em 1927, quando a São Paulo Tramway, Light and Power Company elaborou projeto para a implantação de uma rede de transporte urbano sobre trilhos na cidade. Desde então, diversos projetos foram sucessivamente propostos e abandonados, até que, em 1966, a Câmara Municipal de São Paulo aprovou a Lei no ${ }^{\circ}$ 6.988, de 26.12.1966, que criou a Companhia do Metropolitano de São Paulo. Em seguida, foi realizada uma concorrência internacional para um estudo de viabilidade econômico-financeira, e a empresa vencedora elaborou o projeto de uma rede de $70 \mathrm{~km}$ de vias divididas em quatro linhas. As obras começaram em 1968, e, em 1972, foi inaugurado o primeiro trecho, entre as estações Jabaquara e Saúde. Nos anos seguintes, outros trechos foram construídos e adicionados à rede até atingir os $63 \mathrm{~km}$ de linhas e 60 estações existentes em 2011. Atualmente, a rede é composta por cinco linhas em código de cores: Linha 1 - Azul, Linha 2 - Verde, Linha 3 - Vermelha, Linha 4 - Amarela e Linha 5 - Lilás, transportando 3,3 milhões de passageiros por dia.

A Linha 4 teve seu traçado idealizado nos anos 1940 e, desde então, esteve presente em todos os estudos de ampliação da rede férrea urbana. Ao longo dos anos, seu trajeto sofreu alterações, consolidando-se em 1968 como a Linha Sudeste-Sudoeste, que conectaria os bairros de Pinheiros e Sacomã, passando pelo Centro e cortando a linha Leste-Oeste do Metrô nas estações República e Pedro II. Em 1993, quando o primeiro projeto de construção foi elaborado, o trecho Sudeste foi excluído, incorporando-se outras diretrizes de expansão do Metrô e melhorias dos trens metropolitanos.

Um estudo realizado pelo Governo de São Paulo e pelo International Finance Corporation (IFC) do Banco
Mundial, em 1997, indicou que esse projeto não seria atraente para o investidor privado, devido às grandes incertezas existentes a respeito da demanda projetada (PIONER, 2010). Para viabilizar a implantação dessa classe de projetos, em dezembro de 2004, foi promulgada no Brasil a Lei Federal no 11.079/2004, conhecida como Lei das Parceiras Público-Privadas (PPP), que instituiu normas gerais para licitação e participação governamental em conjunto com o setor privado em projetos de infraestrutura no país. Nessa modalidade de contrato, o risco do projeto pode ser mitigado por meio de alguma forma de apoio governamental, como, entre outros, o pagamento de contraprestações, garantias de demanda, de taxa de câmbio e de retorno do investimento.

Uma forma usual é a concessão de uma garantia de que a demanda não será inferior a um patamar contratual predeterminado: caso o tráfego verificado seja insuficiente para garantir a demanda mínima acordada, o Governo cobre a diferença, caracterizando, dessa forma, um seguro para o concessionário. Por outro lado, na avaliação do Governo paulista, a experiência com o investimento privado em projetos de infraestrutura como as concessões rodoviárias e distribuição de gás natural havia sido muito positiva, gerando um ambiente favorável para a adoção desse modelo no Estado de São Paulo. Dessa forma, optou-se por conceder ao investidor privado a operação da linha e, em 2004, o governador Geraldo Alckmin sancionou a Lei no 11.688 , permitindo que o projeto da Linha 4 fosse executado por meio de uma PPP. O Edital de Concorrência Internacional $\mathrm{n}^{\mathrm{0}} 42325.212$, estabelecendo um prazo de 30 anos (2008 a 2038) para a operação e exploração dos serviços de transporte de passageiros da Linha 4 , mediante a cobrança de tarifa única, foi publicado em dezembro de 2005.

O edital apresentava algumas características inovadoras na história das concessões de infraestrutura no Brasil, na forma de mecanismos de mitigação de riscos, que teriam importantes consequências para a valoração do projeto. No caso da concessão da Linha 4, o Governo ofereceu três tipos de apoio: o pagamento de uma contraprestação pecuniária, uma garantia parcial de taxa de câmbio e uma garantia de demanda que variava entre $10 \%$ e $40 \%$ da demanda projetada, conforme o nível de tráfego observado, assegurando uma divisão do risco do projeto entre a concessionária e o Governo de São Paulo. O vencedor da licitação seria a empresa que ofertasse o menor valor para a contraprestação do Estado. Em termos financeiros, garantias contratuais possuem características de um seguro, ou opção, cujo efeito é 
o de reduzir o risco e, consequentemente, aumentar o valor do projeto. Como a garantia altera o risco do projeto, os métodos tradicionais de análise de projetos de investimento, como o método do Fluxo de Caixa Descontado (FCD), não podem ser utilizados, pois não há como se saber, a priori, qual a taxa de desconto que corresponde a essa redução no risco do projeto. Além disso, no caso da garantia de demanda, o impacto da incerteza de tráfego é assimétrico, pois, enquanto as variações abaixo do valor esperado são irrestritas, as variações para maior são limitadas pela capacidade de escoamento de tráfego da via. Dessa forma, a valoração do projeto exige o uso de métodos de apreçamento de opções, ou opções reais.

A concorrência foi vencida pelo consórcio MetroQuatro, liderado pela Companhia de Concessões Rodoviárias (CCR), que ofereceu uma contraprestação de R\$ 75 milhões, e o primeiro contrato de PPP no Brasil foi assinado em novembro de 2006. Neste trabalho, analisamos a influência dos incentivos oferecidos pelo edital de licitação da Linha 4 do Metrô de São Paulo sobre o projeto por meio da metodologia de opções reais, com o objetivo de verificar qual o nível de redução de risco obtido, bem como o custo e o risco dessas garantias para o Estado. Além disso, utilizamos o modelo desenvolvido neste artigo para determinar a proporção ótima de contraprestação e garantia de demanda que minimiza o custo total para o Governo.

Este trabalho está organizado da seguinte forma: após esta introdução, é feita uma revisão da literatura relevante, e, em seguida, apresentamos, na seção 3, a análise do projeto da Linha 4 pelo método do FCD. Na seção 4, desenvolvemos o modelo de opções reais do projeto, o efeito da limitação de capacidade e propomos uma metodologia para o desenho ótimo dos incentivos e, na seção 5, concluímos.

\section{REVISÃO DA LITERATURA}

A decisão de investimento de capital é uma das principais formas de criação de valor para o acionista, sendo a metodologia do FCD introduzida nas empresas a partir da segunda metade do século XX, a ferramenta mais utilizada para essa finalidade (GRAHAM E HARVEY, 2003). No entanto, o método do FCD ignora o valor da oportunidade de se alterar a estratégia operacional de um projeto em resposta a alterações nas condições de mercado.
Mais recentemente, o trabalho pioneiro de Black e Scholes (1973) e Merton (1973) para o apreçamento de opções financeiras forneceu a base para a incorporação dessa metodologia para projetos em condições de incerteza. Essa metodologia, conhecida como teoria das opções reais para indicar a sua aplicação a opções sobre ativos reais, permite capturar o valor das flexibilidades gerenciais, ou opções, existentes em diversas classes de projetos. Tourinho (1979) foi o primeiro a utilizar o conceito de opções reais para avaliar um reservatório de petróleo com incerteza de preço, enquanto Brenann e Schwartz (1985) analisam estratégias operacionais em uma mina de cobre. McDonald e Siegel (1986) mostram como o momento ótimo para investir em um projeto com incerteza de receitas e custos pode ser determinado, e Dixit e Pindyck (1994) e Trigeorgis (1996) foram os primeiros a sintetizar os principais conceitos dessa metodologia e ilustram a sua aplicação a diversos setores da economia.

A literatura sobre o uso de opções reais para o apreçamento de garantias governamentais em projetos de infraestrutura é extensa. Rose (1998) utiliza o caso da Melbourne Central Toll Project, na Austrália, como exemplo de aplicação e conclui que ignorar as flexibilidades proporcionadas por essas garantias nesse projeto pode resultar na sua subavaliação. Cui e outros (2004) também analisam projetos rodoviários e concluem que garantias são um modo de se prover ao Estado maior flexibilidade gerencial sobre os projetos que envolvem PPPs. Rus e Nombela (2003) mostram que existem duas dificuldades principais para a viabilização de projetos de concessão rodoviária: assimetria de informações de custos, que, normalmente, é resolvida pelo leilão de seleção do concessionário, e risco de demanda. Os autores concluem que a adoção de mecanismos de garantia ao concessionário, ajustando o contrato à demanda real, é a melhor maneira de solucionar tal problema.

Cheah e Liu (2006) utilizam a metodologia das opções reais para analisar o projeto de ampliação da ponte entre a Malásia e Cingapura, onde o Governo ofereceu uma garantia caso o tráfego fosse menor que o previsto, sendo que o concessionário devolveria $\mathrm{o}$ excesso de receita, caso fosse maior. Chiara, Garvin e Vecer (2007) sugerem que o melhor modelo de garantia para um projeto do tipo Build, Operate, Transfer (BOT) é um composto de opções bermudianas, que podem ser exercidas uma única vez em datas predeterminadas, e australianas, que podem ser exercidas $M$ vezes, em $\mathrm{N}$ datas predeterminadas, em que $\mathrm{M} \geq \mathrm{N}$, e concluem que o método dos mínimos quadrados de Longstaff e 
Schwartz (2001) pode ser utilizado para determinar o apreçamento dessas garantias.

Bowe e Lee (2004) analisam o projeto de construção da Taiwan High-Speed Rail Project baseados em informações do consórcio vencedor do projeto e do Governo e considerando as opções de adiamento, expansão, contração e abandono, além de considerar a opção da concessionária de desenvolver projetos imobiliários ao longo da faixa de domínio da concessão. Os autores concluem que essas flexibilidades gerenciais adicionam valor ao projeto e reduzem significativamente o risco. Huang e Chou (2006) analisam o mesmo projeto com enfoque na garantia de receita e a opção de abandono na fase de pré-construção, e concluem que essas opções agregam substancial valor ao projeto. Brandão e Saraiva (2008) também analisam o problema das garantias em PPP, enfocando o caso de concessões rodoviárias, a eficácia das garantias de tráfego na redução do risco do projeto e seus custos para o Governo, e propõem medidas para limitar o passivo e o risco que o Governo assume ao conceder essas garantias. Galera e Solino (2010) utilizam a mesma metodologia desenvolvida em Brandão e Saraiva (2008) para analisar projetos de concessão rodoviária nos quais existam cláusulas de garantias de tráfego, obtendo conclusões semelhantes. Nenhum dos trabalhos encontrados na literatura, no entanto, considera um modelo em que sejam adotados níveis de garantias distintos para cada faixa de tráfego como forma de minimizar o risco da não realização das receitas estimadas, que foi utilizado no caso da L4, nem o impacto que a limitação de capacidade da linha tem sobre o valor e risco do projeto.

\section{MODELO DE FCD DO PROJETO DA LINHA 4}

De acordo com o edital de licitação, o custo do projeto foi orçado em $\mathrm{R}$ \$ 3,34 bilhões, sendo R \$ 2,31 bilhões correspondentes às obras civis, estações e sistemas de controle e comando de responsabilidade do Estado e R $\$ 1,03$ bilhão por parte do investidor privado, referente ao material rodante e operação do sistema, conforme ilustrado na Tabela 1.

Para melhorar a viabilidade econômico-financeira do projeto, foi prevista a sua implantação em duas fases. Na Fase I, seriam construídos 12,8 km de via, seis estações, a estrutura de três estações intermediárias, integração com as demais linhas de Metrô, sistemas e pátio de manutenção, suficientes para a linha entrar em operação gerando receita para o concessionário. A Fase II, que seria implantada quatro anos após o início da Fase I, envolvia a conclusão das estações intermediárias, a construção de duas novas estações e de sistemas complementares.

A concessionária seria ressarcida pelo investimento, manutenção e operação da linha por meio da cobrança de tarifas de uso dos passageiros, contraprestação paga pelo Estado e outras receitas não tarifárias. A receita tarifária seria uma porcentagem da tarifa de remuneração por passageiro transportado, fixada em $\mathrm{R} \$ 2,08$ na data base de $1^{\circ}$ de fevereiro de 2005, também chamada de tarifa de remuneração. Esse valor seria reajustado, nos primeiros 15 anos, pela média ponderada do IGP-M e do IPC, e, após esse prazo, somente pela variação do IPC, até o final da concessão. A concessionária receberia $100 \%$ do valor da tarifa de remuneração vigente dos passageiros que utilizassem exclusivamente a Linha Amarela, sem utilizar outra linha metroferroviária. Já no caso de passageiros integrados ao sistema metroferroviário, a concessionária receberia o valor correspondente a 50\% da tarifa. O Estado também ressarciria à concessionária $100 \%$ das gratuidades, também de acordo com o critério acima. Os reajustes tarifários seriam condicionados ao cumprimento, pela concessionária, dos indicadores de qualidade dos serviços prestados e de manutenção, que foram considerados como uma perda de $2 \%$ da receita ao longo do prazo de concessão.

A contraprestação pecuniária representava o valor que o Governo pagaria à concessionária a título de fundo perdido em parcelas mensais, ao longo dos quatro anos iniciais, que correspondiam à fase das obras, limitado ao valor nominal total de R\$ 120 milhões. O edital

\begin{tabular}{|l|c|c|c|c|}
$\begin{array}{l}\text { Tabela 1 - Investimentos previstos } \\
\text { (R\$ milhões) }\end{array}$ & \multicolumn{3}{|c|}{} \\
\hline \multicolumn{1}{|c|}{ Responsável } & Fase I & Fase II & Total & Prop \\
\hline Governo do Estado & 1.845 & 470 & 2.315 & $69 \%$ \\
\hline Obras Civis & 1.700 & 98 & 1.797 & \\
\hline Estações & 50 & 207 & 258 & \\
\hline Sistemas & 95 & 165 & 260 & \\
\hline Concessionário & 520 & 507 & 1.027 & $31 \%$ \\
\hline Material Rodante & 362 & 467 & 829 & \\
\hline Sistemas & 158 & 40 & 198 & \\
\hline Total & 2.365 & 977 & 3.342 & $100 \%$ \\
\hline
\end{tabular}

Fonte: Edital de Licitação n⿳0 42325.212 (convertido para reais). 
estipulava que o concorrente que solicitasse o menor valor de contraprestação pecuniária seria o vencedor da licitação. O concessionário teria também o direito a obter receitas não tarifárias, referentes a atividades como a exploração de comércio e publicidade nas estações, contanto que não interferissem na qualidade dos serviços. Para efeito da análise do projeto, essas receitas foram estimadas em $5 \%$ das receitas totais.

De acordo com o edital, o Governo seria responsável pelos riscos de construção, obrigando-se a ressarcir o concessionário no caso de eventuais atrasos na obra civil que pudessem retardar o início da operação da linha. Uma possível concorrência entre o transporte público rodoviário e o ferroviário também poderia afetar negativamente o concessionário, cabendo, nesse caso, ao Governo garantir a não competição entre o Metrô e as linhas de ônibus. O parceiro privado, por sua vez, seria responsável pelos riscos relativos à implantação, operação e manutenção dos sistemas sob sua responsabilidade, incluindo atrasos na entrega dos equipamentos e na obtenção dos recursos para os investimentos.

A demanda projetada, estimada com base nas informações constantes do Anexo IV do Edital de Concorrência Internacional no 42325.212 (2005) e Audiência Pública (2005), está ilustrada na Tabela 2, que assume que haverá um crescimento acelerado nos quatro anos iniciais, seguido de um crescimento vegetativo de $1,3 \%$ a.a. a partir de 2012. Esse crescimento irá estabilizar-se em 2020, permanecendo constante a partir daí até o final da concessão, em 2038.

A receita foi determinada levando-se em conta a demanda de passageiros, o preço da passagem e o tipo de passageiro a utilizar a linha (exclusivo ou integrado), e o fator de cumprimento dos indicadores de qualidade dos serviços prestados e de manutenção, estimado em $98 \%$ da receita. De acordo com o edital, a concessionária tem o direito, também, de explorar fontes de receitas alternativas não tarifárias, que foram assumidas como 5\% da receita tarifária total. Os custos foram estimados com base nos critérios definidos no edital, e sua composição envolve os custos operacionais diretos, relativos à operação da linha, custos operacionais indiretos, relativos às despesas de marketing, seguros, garantias, legal, administrativas e outros, e custos de investimento necessários para a execução das Fases I e II. Foram considerados, ainda, os impostos incidentes sobre a receita tarifária (0,65\% PIS e 3\% Cofins), receita não tarifária ( $5 \%$ ISS, 1,65\% PIS e 7,6\% Cofins), além de Imposto de Renda e Contribuição Social sobre o Lucro na alíquota de $30 \%$.

Para o cálculo do WACC, foi utilizado o modelo CAPM, ajustado à taxa de risco do Brasil. Para a taxa livre de risco real $\left(R_{F}\right)$, foi considerado o retorno dos títulos do tesouro do Governo norte-americano de 30 anos na época do lançamento do edital de licitação (4,63\% ao ano, segundo o site do Federal Reserve http://www.federalreserve.gov), descontada a inflação norte-americana projetada de $1 \%$ ao ano, obtendo-se, então, a taxa de $R_{F}=3,63 \%$. O prêmio de risco histórico do mercado norte-americano $(\lambda)$ foi estimado em $8 \%$ a.a., a taxa de Risco Brasil (RB), em 4\% a.a., e o Beta desalavancado $\left(\boldsymbol{\beta}_{U}\right)$, em 0,75 . Para o valor de $\boldsymbol{\beta}_{U}$, foi tomado como referência o Beta setorial de empresas de estrada de ferro norte-americanas $(0,58)$, obtido do site http://pages.stern.nyu.edu/ adamodar/, ajustado para maior para refletir o maior risco de transporte

\begin{tabular}{|c|c|c|c|c|c|c|c|c|c|c|c|}
\hline & 2008 & 2009 & 2010 & 2011 & 2012 & 2013 & 2014 & 2015 & 2016 & 2017 & 2018 \\
\hline Fase I & 308 & 704 & 730 & 739 & & & & & & & \\
\hline \multirow[t]{2}{*}{ Fase II } & & & & & 970 & 981 & 992 & 1.003 & 1.015 & 1.026 & 1.038 \\
\hline & & 2019 & 2020 & 2021 & 2022 & 2023 & 2024 & 2025 & 2026 & 2027 & 2028 \\
\hline \multirow[t]{2}{*}{ Fase II } & & 1.049 & 1.061 & 1.061 & 1.061 & 1.061 & 1.061 & 1.061 & 1.061 & 1.061 & 1.061 \\
\hline & & 2029 & 2030 & 2031 & 2032 & 2033 & 2034 & 2035 & 2036 & 2037 & 2038 \\
\hline Fase II & & 1.061 & 1.061 & 1.061 & 1.061 & 1.061 & 1.061 & 1.061 & 1.061 & 1.061 & 1.061 \\
\hline
\end{tabular}

Fonte: Edital de Licitação nº 42325.212. 
urbano. Para o cálculo do Beta alavancado $\left(\beta_{L}\right)$, foi feita a transformação ilustrada na Eq. (1) considerando uma alíquota efetiva de IR de 30\%, e relação $\operatorname{Debt}(D) /$ Equity (E) de 40\%/60\%, obtendo-se o valor de $\beta_{L}=1,1$.

$$
\beta_{L}=\beta_{U} x\left[1+(1-I R) x\left(\frac{D}{E}\right)\right]
$$

Dessa forma, por meio do modelo CAPM $K_{e}=R_{F}$ $+B_{L} \lambda+R_{B}$, obtemos a taxa de $K_{e}=16,43 \%$ a.a., que representa a expectativa do investidor à época da participação da concorrência e tomada de decisão de investimento (dezembro de 2005). Considerando um nível de endividamento geral de $40 \%$ e custo bruto de capital de terceiros de $13,83 \%$, obtém-se um custo médio de capital ponderado real de capital (WACC) de 12,38\%. A análise feita por meio da metodologia do FCD resulta em um VPL de R 151,8 milhões (incluindo a contraprestação nominal de $\mathrm{R} \$ 75$ milhões), e uma TIRM de 13,38\%. O VPL, sem a contraprestação, é de R\$ 108,2 milhões.

\section{ANÁLISE POR OPÇÕES REAIS}

A análise estática do FCD não permite uma avaliação mais precisa dos riscos envolvidos nem captura o valor das garantias de mitigação de risco oferecidas no edital, que, por se configurarem como um seguro, somente podem ser avaliadas por meio de métodos de apreçamento de opções. De acordo com o edital de concorrência, os riscos relativos à variação cambial e demanda de tráfego seriam compartilhados pelo concessionário e Governo. Considerando que uma parte significativa dos equipamentos seria importada, uma vez que a tecnologia para a construção desse tipo de trem ainda não era inteiramente dominada pela indústria nacional, uma variação acentuada na taxa de câmbio poderia afetar a capacidade de pagamento dos compromissos em moeda estrangeira, já que a totalidade da receita do projeto é auferida em reais. Para mitigar esse risco, o Governo arcaria com até 50\% desse custo, caso solicitado pela concessionária.

O risco de tráfego está associado à possibilidade de que a demanda projetada não se verifique na prática e configura-se tradicionalmente como o principal risco dessa classe de projetos. No caso da Linha 4, para mitigar esse risco, o edital previa um mecanismo inédito no Brasil de concessão de garantias de demanda escalonadas por faixas. Esse mecanismo entraria em vigor seis meses após o início da operação comercial da Fase I e teria duração de 10 anos (2008 a 2018). As regras de mitigação estabeleciam que o concessionário receberia uma compensação $(M)$ sempre que a demanda real verificada no trimestre $(D R)$ ficasse abaixo de $90 \%$ da demanda projetada para o período $(D P)$, até o limite de 60\%. Caso a demanda ficasse abaixo de 60\% do projetado, caberia a recomposição do equilíbrio econômico financeiro do contrato em favor do concessionário. Da mesma forma, caso $D R>110 \% D P$, o concessionário repassaria parte desse ganho para o Governo. O valor da mitigação $M$ dependeria da faixa de demanda observada, conforme ilustrado na Tabela 3 , sendo que, para variações de demanda menores do que $\pm 10 \%$ da demanda projetada, não haveria qualquer compensação. Os valores de mitigação seriam, ainda, ponderados pela proporção de passageiros exclusivos da Linha 4 em relação ao total de usuários.

A principal incerteza no projeto da Linha 4 do Metrô de São Paulo está relacionada com o número de usuários que irão demandar seus serviços. Para a mo-

\section{Tabela 3 - Garantia de demanda}

\begin{tabular}{|c|c|c|}
\hline Demanda real (DR) & Valor da mitigação (M) & Favorecido \\
\hline $90 \% D_{P}<D_{R} \leq 110 \% D_{P}$ & $M=0$ & - \\
\hline $80 \% D_{P}<D_{R} \leq 90 \% D_{P}$ & $M=0,6\left(0,90 \times D_{P}-D_{R}\right)$ & Concessionário \\
\hline $60 \% D_{P}<D_{R} \leq 80 \% D_{P}$ & $M=0,06 \times D_{P}+0,9\left(0,80 \times D_{P}-D_{R}\right)$ & Concessionário \\
\hline$D_{R}<60 \% D_{P}$ & Recomposição financeira do contrato & Concessionário \\
\hline $110 \% D_{P}<D_{R} \leq 120 \% D_{P}$ & $M=0,6\left(D_{R}-1,1 \times D_{P}\right)$ & Governo \\
\hline $120 \% D_{P}<D_{R} \leq 140 \% D_{P}$ & $M=0,06 \times D_{P}+0,9\left(D_{R}-1,2 \times D_{P}\right)$ & Governo \\
\hline$D_{R}>140 \% D_{P}$ & Recomposição financeira do contrato & Governo \\
\hline
\end{tabular}


delagem dessa incerteza, como é padrão na literatura, assumimos que o tráfego projetado varia de maneira estocástica, seguindo o Movimento Geométrico Browniano (MGB), conforme a equação (2):

$d S=\mu S d t+\sigma S d z$

onde:

$d S=$ variação incremental do tráfego no intervalo de tempo $\Delta t$

$\mu=$ taxa de crescimento esperado do tráfego

$\sigma=$ volatilidade do tráfego

$d z=\varepsilon \sqrt{d t}$ é o incremento de Wierner padrão

A modelagem estocástica do tráfego é feita discretizando-se esse processo em intervalos de tempo anuais de acordo com a equação (3):

$$
S_{t+1}=S_{t} e^{\left(\mu-\frac{\sigma^{2}}{2}\right) \Delta t+\sigma \varepsilon \sqrt{\Delta t}}
$$

A taxa esperada de crescimento do tráfego $(\boldsymbol{\mu})$ ao longo da vida do projeto é conhecida, e já foi utilizada no cálculo do VPL. Assumimos, também, que existe uma incerteza a respeito do nível de tráfego inicial do projeto, que foi modelado como uma distribuição triangular com valores mínimo e máximo de 215.000 e 400.000, respectivamente, e esperado de 308.000. Para a determinação do parâmetro da volatilidade $(\sigma)$, para projetos rodoviários, é usual utilizar-se como referência o desvio padrão dos log retornos do PIB regional
(IRWIN, 2007), uma vez que há uma forte correlação entre tráfego rodoviário e crescimento econômico. A série histórica deflacionada do PIB da cidade de São Paulo de 1999 a 2008 indica uma volatilidade de 3,9\% a.a. No entanto, o tráfego rodoviário é fortemente influenciado pelo transporte de carga, o que não é o caso do Metrô, cuja demanda é essencialmente de passageiros. Por outro lado, a série histórica de viagens de Metrô em direção ao Centro em São Paulo entre 1977 a 1987 (PIOVEZAN, 1991) apresenta uma volatilidade significativamente maior, de $15,74 \%$. Para fins deste trabalho, assumimos uma volatilidade de $8 \%$ e realizamos uma análise de sensibilidade para valores entre $4 \%$ e $16 \%$ ao final.

A análise dinâmica do projeto básico mostra que existe uma probabilidade de $34,8 \%$ de o VPL ser negativo, o que indica que a oferta de modos de mitigação de risco para o investidor privado é adequada. Considerando uma contraprestação nominal de $\mathrm{R} \$$ 75 milhões, equivalente ao valor solicitado pelo consórcio vencedor da licitação, o VPL aumenta para R\$ 151,8 milhões e o risco de VPL negativo reduz-se para 27,2\% (Figura 1).

Os mecanismos de mitigação de risco estabelecidos no edital de licitação da Linha 4 tiveram por objetivo aumentar a atratividade do projeto para os investidores privados, conforme previsto na Lei das PPPs. Esse efeito é obtido pela concessão de garantias de demanda mínima, que tem efeito semelhante ao de um seguro para o concessionário. Por alterarem o risco do projeto, o cálculo do valor e do impacto dessas garantias não

\section{Figura 1 - Análise do risco de demanda com e sem contraprestação de R\$ 75 milhões}

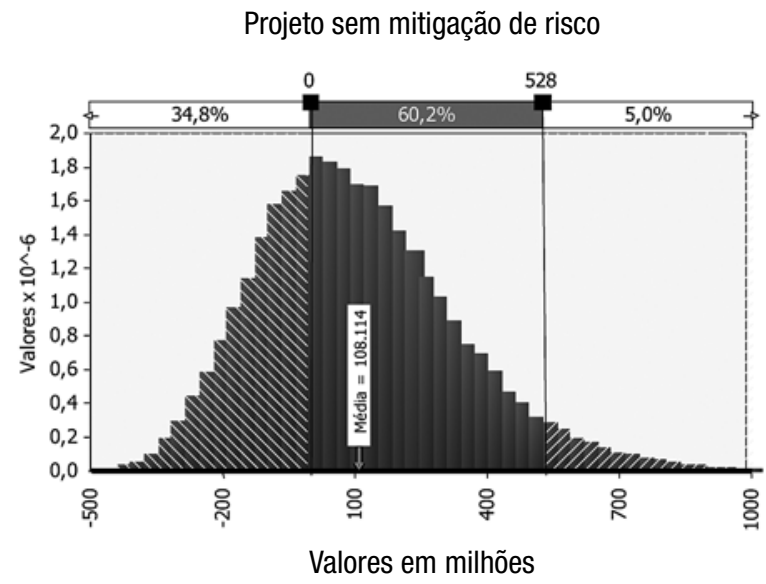

Projeto com contraprestação

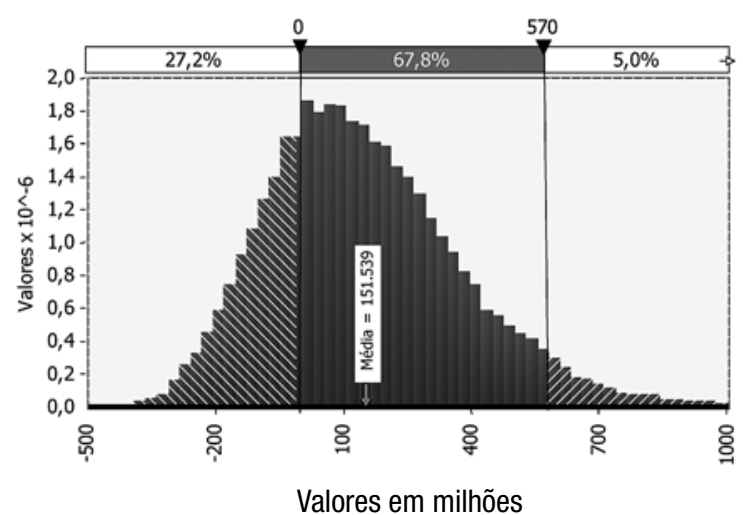

Valores em milhões 
pode ser realizado por meio do FCD tradicional, uma vez que não há como determinar, a priori, qual a taxa de desconto a ser utilizada para o novo nível de risco do projeto. Dessa forma, para analisar o impacto desses mecanismos de mitigação, é utilizada a metodologia de opções reais.

Para essa análise, foram adotadas algumas premissas simplificadoras. Embora o edital de licitação estabeleça que a mitigação será trimestral, nos nossos cálculos, adotamos periodicidade anual. Essa premissa é conservadora, uma vez que considera que eventuais pagamentos do Estado ao concessionário ocorrerão apenas ao final de cada ano, e não trimestralmente. O mesmo raciocínio aplica-se aos pagamentos do concessionário para o Governo, mas veremos que estes são significantemente menores. Foi desconsiderada também a parcela (50\%) do risco cambial de responsabilidade do concessionário. O edital estipula, ainda, que, caso a demanda real seja menor do que $60 \%$ ou maior do que $140 \%$ da demanda projetada, haverá uma renegociação do contrato. Considerou-se que uma eventual renegociação necessariamente resultará em ônus adicionais para o Estado, uma vez que os serviços não poderão ser interrompidos, e que a hipótese de uma renegociação favorável ao Estado é inexistente, uma vez que a limitação de capacidade de passageiros na linha assegura que níveis acima de $140 \%$ da demanda esperada nunca serão atingidos. Dessa forma, neste trabalho, esse limite foi ignorado e as regras de mitigação, aplicadas a todos os intervalos de demanda possíveis.

As garantias de demanda foram analisadas como uma sequência de opções europeias com vencimentos em cada ano do projeto, uma vez que são independentes entre si (Charoenpornpattana, Minato, Nakahama, 2003). As garantias de demanda mínima são modeladas como opções de venda (Put) em favor do concessionário, em que o concessionário tem o direito de receber a diferença entre a demanda real e a demanda garantida, caso esta seja maior. Da mesma forma, a obrigação de repasse ao Governo da demanda excedente é modelada como opções de compra (Call) em favor do Estado. Para cada um desses anos, são modelados dois níveis de garantia acima e abaixo do valor de tráfego esperado, dependendo de ser a garantia a favor do concessionário ou do Governo, e somente um desses casos pode ocorrer em qualquer ano particular, ou nenhum, caso o tráfego apresente variação menor que $10 \%$ em relação ao projetado. No caso de o tráfego ser acima do previsto, o Governo tem a opção de receber as receitas excedentes.
A opção do concessionário, isoladamente, pode ser modelada como uma opção de venda (Put) europeia conforme Eq. (4), em que $D(t)$ é a demanda final recebida no ano $t, D_{R}(t)$ é a demanda real observada e $M(t)$ é o valor da mitigação devida naquele ano.

$$
D(t)=\max \left[D_{R}(t), D_{R}(t)+M(t)\right]
$$

Por outro lado, o edital estabelece que, caso o tráfego real observado seja significativamente maior do que o previsto, o concessionário deverá repassar parte desse excesso para o Governo. Ambas as opções (Call e Put) precisam ser modeladas simultaneamente como um caso de opções compostas, uma vez que ambas são exercidas sobre o mesmo ativo básico, a demanda de tráfego. A modelagem dessas opções deve ser feita considerando-se que o real nível de tráfego pode estar em cinco regiões distintas e mutuamente exclusivas, conforme ilustrado na Tabela 3. Nesse caso, a regra do valor ótimo do projeto, em cada período $t$, é dada pela Eq. (5)

$$
D(t)=\min \left\{\max \left[D_{R}(t), D_{R}(t)+M(t)\right], D_{R}(t)-M(t)\right\}(5)
$$

O apreçamento das opções foi realizado por meio de simulação de Monte Carlo, utilizando-se a medida neutra a risco, com o processo descrito pela equação $d S_{R}=(\mu-\delta) S_{R} d t+\sigma S_{R} d z$, em que $\mu$ é a taxa de crescimento e $\delta$ é o prêmio de risco do tráfego. Como os mercados são incompletos para o risco de tráfego, a determinação do prêmio de risco somente pode ser feita por métodos indiretos. Para tanto, adotou-se o método proposto por Freitas e Brandão (2010) de igualar os fluxos de caixa tradicional e neutro a risco do projeto, conforme equação (6)

$$
\sum_{i=1}^{n} \frac{E[f(S)]}{(1+\mu)^{i}}=\sum_{i=1}^{n} \frac{E\left[f\left(S_{R}\right)\right]}{(1+r)^{i}}
$$

em que $d S=\mu S d t+\sigma S d t, d S_{R}=(\mu-\delta) S_{R} d t+\sigma S_{R} d z$ e $f(\cdot)$ é o fluxo de caixa do projeto, obtendo-se um valor de $\delta=2,308 \%$. O projeto foi, em seguida, analisado por meio de uma avaliação neutra a risco com simulação de Monte Carlo, considerando o recebimento da contraprestação pecuniária de $\mathrm{R} \$ 75$ milhões e as flexibilidades gerenciais representadas pelos mecanismos de mitigação de risco descritos na Tabela 3. 
Os resultados para o concessionário indicam que os mecanismos de mitigação de risco definidos no edital de licitação aumentam o VPL do projeto em $35,6 \%$, para $\mathrm{R} \$ 206,6$ milhões, ao mesmo tempo que reduzem de $27,2 \%$ para $16,3 \%$ o risco de que o projeto tenha um retorno negativo. Estendendo-se, por hipótese, o prazo de vigência da mitigação de risco para 20 anos e 30 anos, esse valor sobe para $R \$ 303,7$ milhões e 402,1 milhões, respectivamente, indicando que o projeto é bastante sensível ao prazo de vigência das garantias. Esse aumento significativo ocorre devido ao fato de que, quanto mais no futuro, maior a incerteza a respeito do nível de tráfego que prevalecerá na época, o que torna mais valiosas as garantias governamentais. Isso indica que o Estado de São Paulo foi prudente ao limitar a concessão das garantias de demanda a 10 anos apenas. A Tabela 4 apresenta os resultados da sensibilidade para o parâmetro volatilidade considerando o caso base, em que podemos observar que, como esperado, o valor das opções de garantia aumenta com a volatilidade.

Por outro lado, o aumento do valor do projeto e a redução de risco obtida por meio dos mecanismos de mitigação de risco acarretam um custo para o Estado na forma de um passivo contingente, que, em média, equivale ao valor adicional bruto a ser recebido pelo concessionário. Na Figura 2, podemos

\section{Tabela 4 - Sensibilidade à volatilidade}

\begin{tabular}{|c|c|}
\hline Volatilidade & $\begin{array}{c}\text { VPL } \\
\text { (R\$ 1.000,00) }\end{array}$ \\
\hline $4 \%$ & 190.885 \\
\hline $8 \%$ & 206.611 \\
\hline $12 \%$ & 213.629 \\
\hline $16 \%$ & 221.606 \\
\hline
\end{tabular}

observar que o custo esperado para o Estado das garantias ofertadas é de $\mathrm{R} \$ 88,6$ milhões, sendo que existe uma probabilidade de $5 \%$ de esse valor superar $\mathrm{R} \$ 281,8$ milhões. Por outro lado, o valor esperado dos recebimentos pelo Estado devido ao excesso de tráfego é de $\mathrm{R} \$ 12,3$ milhões, o que mostra que, embora o mecanismo de mitigação seja simétrico, os seus resultados não o são. Isso ocorre porque, como a demanda de tráfego segue um processo de difusão geométrico browniano, a sua distribuição de probabilidade é lognormal, que é assimétrica em relação à média.

\section{Limite de capacidade}

A capacidade de transporte de passageiros na Linha 4

\section{Figura 2 - Distribuição de probabilidade dos custos das garantias para o Estado}

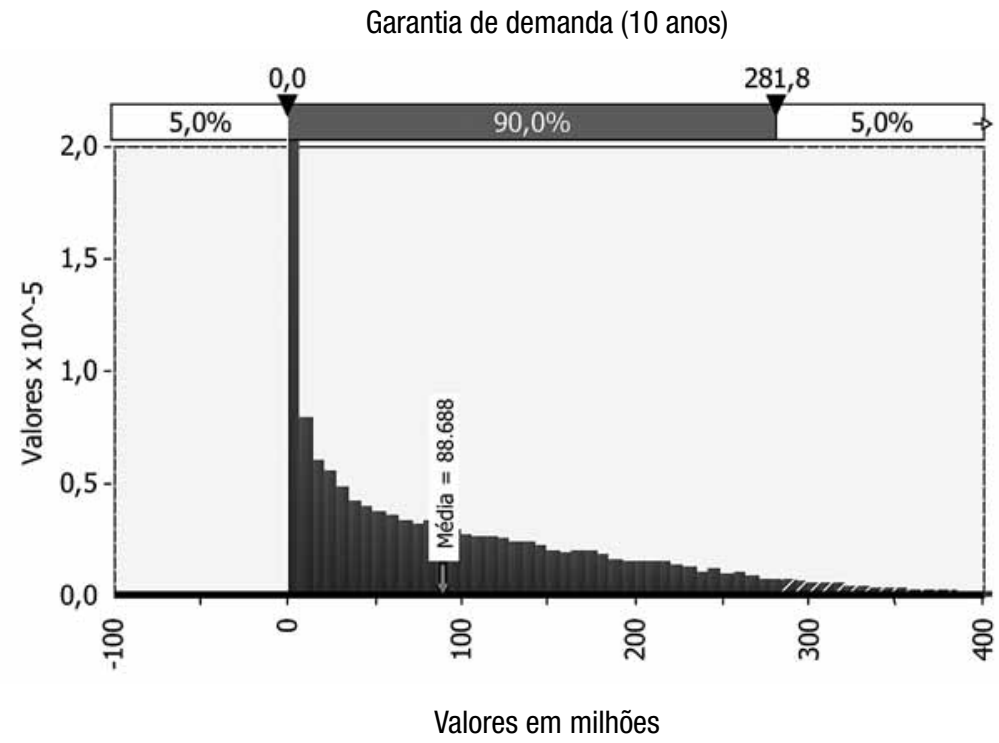


é limitada pelos investimentos em material rodante e infraestrutura de estações. Considerando que é pouco provável que ocorra excesso de capacidade nos anos iniciais, que a fabricação e entrega de material rodante adicional pode demorar vários anos e que a garantia de demanda se encerra em 2018, assumimos que, caso a demanda real se mostre muito acima da demanda projetada, o sistema não terá capacidade de absorver esse excesso de passageiros.

Dessa forma, modelamos limites de capacidade como barreiras 30\%, 20\% e 10\% acima dos níveis de tráfego originalmente projetados. A introdução dessas barreiras de capacidade impõe uma limitação assimétrica na projeção estocástica da demanda, limitando os valores mais altos, acima da barreira, mas sem causar impacto nos valores mais baixos. Isso faz com que o valor esperado da demanda futura sofra uma redução em relação aos valores anteriormente projetados. O valor líquido do projeto com e sem as garantias de demanda está ilustrado na Tabela 5. Em todos os casos, foi considerado o pagamento da contraprestação pecuniária de R 75 milhões. Essas limitações de capacidade também afetam negativamente a expectativa de recebimento de valores por parte do Estado, uma vez que, mesmo que ocorram, demandas muito acima da projetada não serão absorvidas pelo sistema, devido ao congestionamento da linha.

\section{Tabela 5 - Impacto do limite de capacidade}

\begin{tabular}{|l|c|c|}
\hline \multicolumn{1}{|c|}{ Situação } & \multicolumn{2}{|c|}{ VPL (R\$ 1.000,00) } \\
\cline { 2 - 3 } & Sem garantia & Com garantia \\
\hline Caso base, sem barreira & 151.789 & 206.611 \\
\hline Com barreira de 30\% & 147.220 & 203.891 \\
\hline Com barreira de 20\% & 142.856 & 200.851 \\
\hline Com barreira de 10\% & 130.171 & 194.377 \\
\hline
\end{tabular}

Na Figura 3, podemos observar a distribuição de probabilidades de diversos níveis de barreira considerando os mecanismos de mitigação de risco estabelecidos no edital.

\section{Desenho ótimo de mecanismo de mitigação de risco de demanda}

Os incentivos adotados no edital de licitação resultaram num custo, para o Estado, de uma contrapres- tação pecuniária de R 75 milhões e uma garantia de demanda cujo valor presente esperado, ao longo dos seus 10 anos de vigência, é de R \$ 88,7 milhões. Assumindo-se a premissa de neutralidade a risco, ou seja, que o Governo é indiferente entre pagar uma contraprestação firme ou conceder uma garantia de demanda de mesmo valor, o custo total desse mecanismo é de $\mathrm{R} \$ 163,7$ milhões, o que corresponde a $5 \%$ do custo total do projeto ou $16 \%$ do total investido pelo parceiro privado.

Utilizando o modelo desenvolvido neste trabalho, analisamos outras combinações de contraprestação e garantia de demanda, a fim de verificar se haveria outros modelos de incentivo mais eficientes do ponto de vista da redução de risco. Para tanto, analisamos quatro modelos adicionais, todos com o mesmo custo para o Estado, com proporções de contraprestação e garantia variando entre 0 e 100\%, conforme ilustrado na Figura 4.

Foi calculado o custo de garantias de demanda para períodos de sete, 12 e 15 anos, correspondente aos modelos B, C e D, e determinou-se o valor da respectiva contraprestação de modo que o custo total para o Estado seja constante em R \$ 163,7 milhões. Para o modelo $\mathrm{D}$, como o valor da garantia excede esse limite, foi considerada uma contraprestação negativa como compensação. O valor do projeto para cada conjunto de garantia e contraprestação foi calculado utilizando-se a medida neutra a risco, e, em seguida, gerou-se a distribuição de probabilidade de cada modelo, utilizando-se a taxa ajustada ao risco para cada caso. Os resultados estão apresentados na Tabela 6 , na qual podemos observar que, embora o VPL do projeto se mantenha aproximadamente constante, o risco para o concessionário reduz-se à medida que aumenta a parcela referente à garantia de demanda.

\section{CONCLUSÕES}

Neste artigo, analisamos os mecanismos de incentivo para mitigação de risco estabelecidos no Edital de Licitação no 42325.212 para a construção, operação e exploração da Linha 4 do Metrô de São Paulo pelo setor privado, com o objetivo de determinar os impactos desses incentivos sobre o valor e o risco do projeto, bem como o seu custo para o Estado. Um dos componentes desse mecanismo foi uma garantia de demanda, que transfere para o Estado parte do risco de o tráfego futuro de passageiros ser menor 
do que o projetado. Essa forma de garantia tem características de opções cujo valor não pode ser determinado pelos métodos tradicionais de avaliação. Para essa análise, utilizamos a metodologia de opções reais e mostramos como esse modelo de valoração pode ser construído.

Os resultados obtidos indicam que os incentivos propostos são eficazes para reduzir o risco do projeto. O pagamento de contraprestação de $\mathrm{R} \$ 75$ milhões pelo Estado reduz o risco do projeto de $34,8 \%$ para $27,2 \%$, e a garantia de demanda reduz ainda mais esse risco, para $16,3 \%$. Por outro lado, o resultado final do projeto para o concessionário aumenta de $\mathrm{R} \$ 108,2$ milhões para $\mathrm{R} \$ 151,8$ milhões com o pagamento da contraprestação, e para R \$206,6 milhões quando se inclui a garantia de demanda de tráfego, ou seja, um aumento de 36\%. Essa garantia tem um custo esperado para o Estado de R $\$ 88,7$ milhões, com um risco de $5 \%$ de ser maior que $\mathrm{R} \$ 281,8$ milhões. Mostramos, também, que o impacto da garantia de demanda e da limitação de capacidade de tráfego de passageiros na linha é assimétrico, o que faz com que a probabilidade de o Governo do Estado receber pagamentos da concessionária por excesso de passageiros seja pequena.

O modelo de valoração foi aplicado, também, a diferentes modelos de mecanismos de mitigação de risco, mantendo-se constante o custo total para o Estado, considerando-se níveis distintos de participação da contraprestação e da garantia de demanda. Observa-se que o valor do projeto se mantém praticamente constante em cada um desses modelos, mas que o impacto sobre a redução de risco aumenta com o aumento da proporção da garantia no custo total do mecanismo.

\section{Figura 3 - Distribuição de probabilidades com e sem barreiras}

Projeto com garantias e sem barreiras

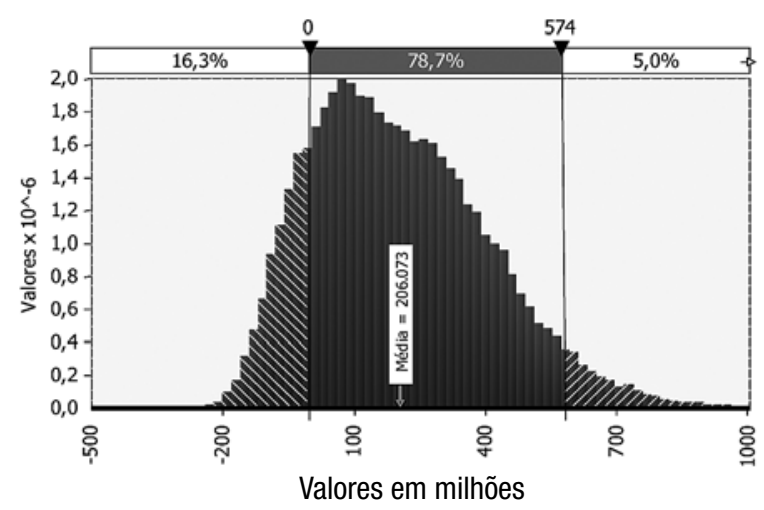

Projeto com garantia e barreira de $20 \%$

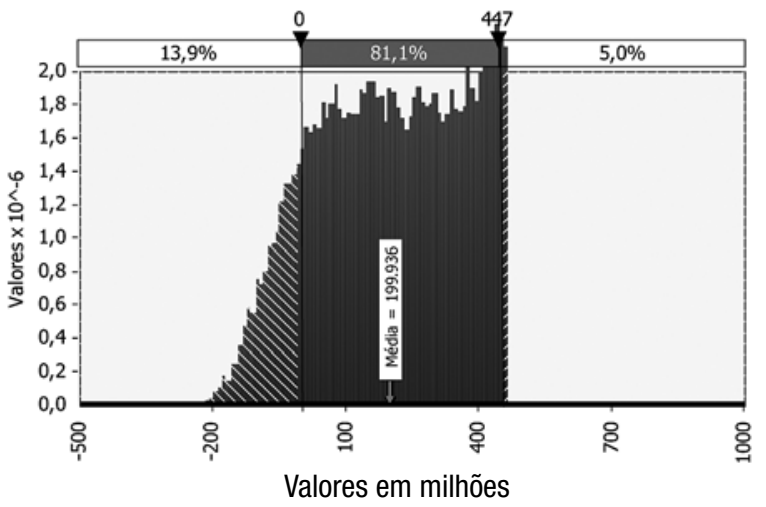

Projeto com garantia e barreira de $30 \%$

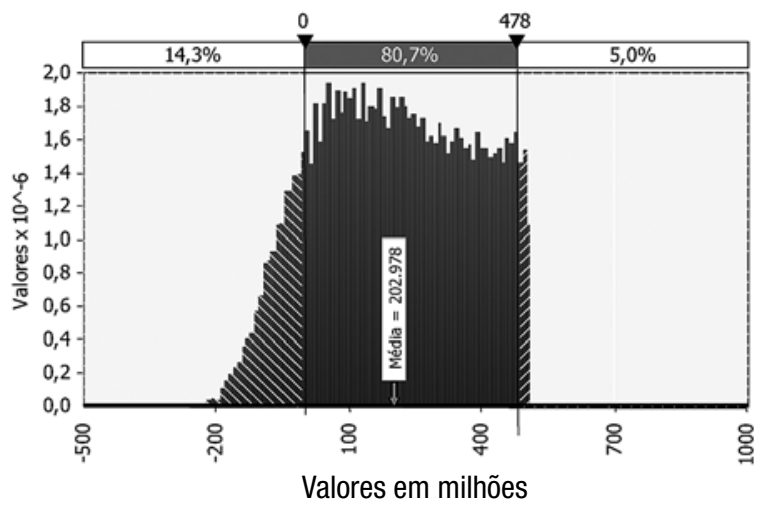

Projeto com garantia e barreira de $10 \%$

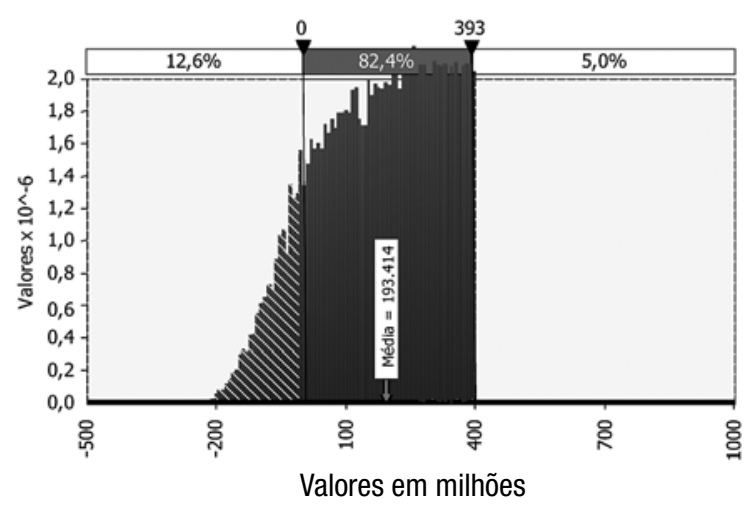


Esse resultado sugere que, para um determinado nível de redução de risco desejado para o projeto, a alternativa de menor custo para o Estado é atingir esse objetivo, aumentando a garantia de demanda e reduzindo o valor da contraprestação.

A metodologia utilizada neste trabalho pode ser aplicada a outros problemas de valoração de PPP em que exista a necessidade de desenhar mecanismos ótimos de mitigação de risco e definir o custo desses mecanismos para o Governo. Cabe notar, no entanto, que a principal utilidade desse tipo de análise reside no fato de que permite tanto ao poder concedente quanto ao investidor privado desenvolver uma sensibilidade e um melhor entendimento dos riscos e custos envolvidos na concessão de garantias contratuais, quando elas se fazem necessárias para a viabilização de projetos de infraestrutura de interesse da sociedade.

As principais limitações desse trabalho dizem respeito à determinação da volatilidade do projeto, cujo

\section{Figura 4 - Modelos alternativos de incentivo}

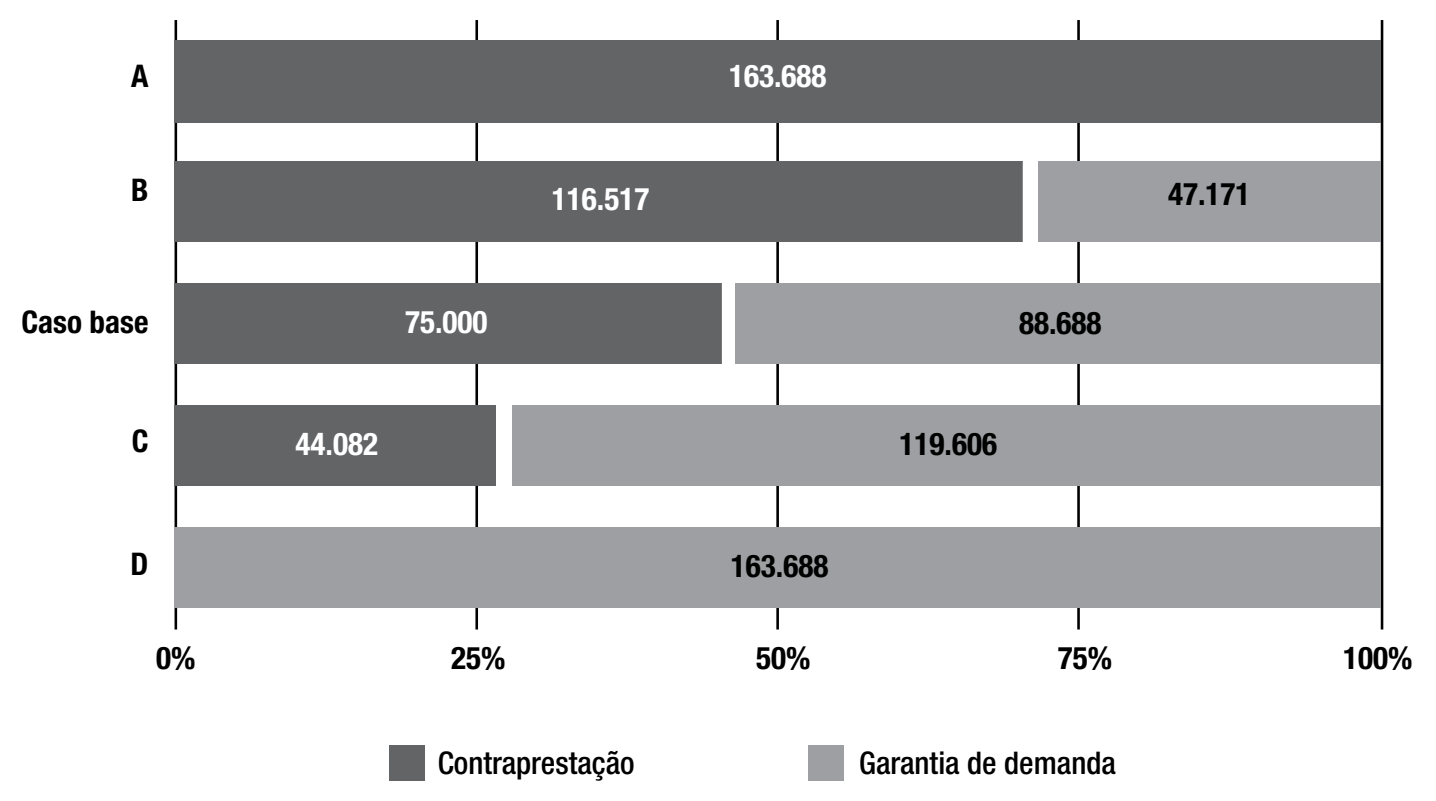

\section{Tabela 6 - Resultado dos modelos alternativos de mitigação}

\begin{tabular}{|l|c|c|c|c|c|}
\hline \multicolumn{1}{|c|}{ R\$ 1.000 } & A & B & Caso base & C & D \\
\hline Duração da garantia (anos) & 0 & 7 & 10 & 12 & 15 \\
\hline & & & & & \\
\hline & 0 & 47.171 & 88.688 & 119.606 & 167.121 \\
\hline Valor da garantia & 163.688 & 116.517 & 75.000 & 44.082 & -3.433 \\
\hline Valor da contraprestação & $\mathbf{1 6 3 . 6 8 8}$ & $\mathbf{1 6 3 . 6 8 8}$ & $\mathbf{1 6 3 . 6 8 8}$ & $\mathbf{1 6 3 . 6 8 8}$ & $\mathbf{1 6 3 . 6 8 8}$ \\
\hline Custo total & & & & & \\
\hline VPL & $\mathbf{2 0 3 . 0 7 4}$ & $\mathbf{2 0 8 . 0 3 4}$ & $\mathbf{2 0 6 . 6 1 1}$ & $\mathbf{2 0 5 . 7 0 6}$ & $\mathbf{2 0 5 . 7 0 7}$ \\
\hline Probabilidade VPL < & $\mathbf{1 9 , 4 0 \%}$ & $\mathbf{1 7 , 1 0 \%}$ & $\mathbf{1 6 , 4 0 \%}$ & $\mathbf{1 5 , 7 0 \%}$ & $\mathbf{1 4 , 6 0 \%}$ \\
\hline
\end{tabular}


valor real somente poderá ser determinado depois do projeto implantado, e à não consideração dos efeitos da garantia cambial, que poderia aumentar os custos para o Estado. Extensões deste trabalho poderiam incluir o estudo do desenho ótimo de incentivos considerando aversão a risco do Governo.

\section{NOTA DE AGRADECIMENTO}

Os autores agradecem o auxílio da FAPERJ através do projeto PRONEM para este trabalho.

\section{REFERÊNCIAS}

BLACK, F; SCHOLES, M. The pricing of options and corporate liabilities. The Journal of Political Economy, v. 81, n. 3, p. 637-654, 1973.

BOWE, M; LEE, D. L. Project evaluation in the presence of multiple embedded real options: evidence from the Taiwan High-Speed Rail Project. Journal of Asian Economics, v. 15, n. 1, p. 71-98, 2004.

BRANDÃO, L. E. T; SARAIVA, E. C. G. The option value of government guarantees in infrastructure projects. Construction Management and Economics, v. 26, n. 11, p. 1171-1180, 2008.

BRENNAN, M. J; SCHWARTZ, E. S. Evaluating natural resource investments. The Journal of Business, v. 58, n. 2, p. $135-157,1985$.

CHAROENPORNPATTANA, S; MINATO, T; NAKAHAMA, S. Government supports as bundle of real options in built-operate-transfer highways projects. 7th Annual International Conference on Real Options. Washington D.C: Real Options Group, 2003.

CHEAH, C. Y. J; LIU, J. Valuing governmental support in infrastructure projects as real options using Monte Carlo simulation. Construction Management and Economics, v. 24, n. 5, p. 545-554, 2006.

CHIARA, N. S. M; GARVIN, J; VECER, J. Valuing simple multiple-exercise real options in infrastructure projects. Journal of Infrastructure Systems, v. 13, n. 2, p. 97-104, 2007.
CUI, Q. e outros. Use of warranties on highway projects: a real option perspective. Journal of Management in Engineering, v. 20, n. 3, p. 118-126, 2004.

DAMODARAN, A. The Data Page. Disponível em: http:// pages.stern.nyu.edu/ adamodar/. Acesso em 17.11.2005.

DIXIT, A. K; PINDYCK, R. S. Investment under uncertainty. Princeton: Princeton University Press, 1994.

FEDERAL RESERVE. Economic Research Data. Disponível em: http://federalreserve.gov. Acesso em 17.11.2005.

FREITAS, A; BRANDÃO, L. Real options valuation of e-learning projects. International Journal on E-Learning, v. 9, n. 3, p. 363-383, 2010.

GALERA, A. L. L; SOLINO, A. S. A Real options approach for the valuation of highway concessions. Transportation Science, v. 44, n. 3, p. 416-427, 2010.

GRAHAM, J. R; HARVEY, C. R. How do CFOs make capital budgeting and capital structure decisions Journal of Applied Corporate Finance, v. 15, n. 1, p. 8-23, 2003.

HUANG, Y. L; CHOU, S. P. Valuation of the minimum revenue guarantee and the option to abandon in BOT infrastructure projects. Construction Management and Economics, v. 24, n. 5, p. 379-389, 2006.

IRWIN, T. C. Government guarantees: allocating and valuing risk in privately financed infrastructure projects. Washington D.C.: The World Bank, 2007. (Directions in development).

LONGSTAFF, F. A; SCHWARTZ, E. S. Valuing American options by simulation: a simple least-squares approach. Review of Financial Studies, v. 14, n. 1, p. 113-147, 2001.

MCDONALD, R; SIEGEL, D. The value of waiting to invest. The Quarterly Journal of Economics, v. 101, n. 4, p. 707-728, 1986.

MERTON, R. C. Theory of rational option pricing. Bell Journal of Economics and Management Science, v.4 (1), p. 141-183, 1973.

PIONER, H. Regulação de transporte. Estudo de caso: PPP da Linha 4 do Metrô de São Paulo. Portal de gestão de conteúdo do programa de parceria público-privada de 
Minas Gerais. Curso de regulação econômica aplicado a permissões, concessões tradicionais, concessões patrocinadas \& administrativas. Belo Horizonte: 25.1.2011. 2010. (Apresentação).

PIOVEZAN, L. H. Viagens ao Centro Velho de São Paulo. São Paulo: CET - Companhia de Engenharia de Tráfego, 1991.

ROSE, S. Valuation of interacting real options in a tollroad infrastructure project. The Quarterly Review of Economics and Finance, v. 38, n. 3, Part 2, p. 711-723, 1998.

RUS, G. D; NOMBELA, G. Variable-term concessions for road construction and operation. Munich: University Library of Munich, 2003. (Unpublished work).

TOURINHO, O. A. F. The valuation of reserves of natural resources: an option pricing approach. 1979. 103p. Tese de Doutorado em Economia. University of California, Berkeley, 1979.

TRIGEORGIS, L. Real options, managerial flexibility and strategy in resources allocation. Cambridge: MIT Press, 1996. 